\title{
Complexos convectivos de mesoescala sobre o Nordeste do Brasil e fenômenos adversos associados
}

A previsão dos Complexos Convectivos de Mesoescala (CCM) tem extrema importância para a população da região Nordeste do Brasil (NEB), pois provocam vários fenômenos adversos como precipitações intensas e trovoadas, consequentemente causando enchentes, alagamentos e deslizamento de terra, trazendo perdas sociais e econômicas. O principal objetivo do presente estudo é analisar a formação dos CCM e seus fenômenos adversos associados para o período entre 2013 e 2015. A identificação dos casos foi feita através da análise de imagens do satélite GOES-13 no canal infravermelho realçado. Dados de reanálise II do National Centers for Environmental Prediction (NCEP) foram utilizados para análise sinótica através dos campos de linhas de corrente com o auxílio do software GrADS. O estudo termodinâmico foi feito através da plotagem de perfis verticais simulados da atmosfera, com a análise baseada nos índices de instabilidade LI e CAPE+. Imagens do satélite Tropical Rainfall Measuring Mission (TRMM) e dados do Departamento de Controle do Espaço Aéreo (DECEA) foram utilizados para a detecção de trovoadas. $O$ registro de precipitação foi coletado das estações automáticas e convencionais da rede de postos pluviométricos do Instituto Nacional de Meteorologia (INMET). Durante 0 período de estudo foram identificados 16 casos de CCM, com registro de trovoada associada em 6 eventos. Quatro diferentes padrões sinóticos foram associados à formação dos CCM Vórtice Ciclônico em Altos Níveis (VCAN), Zona de Convergência Intertropical (ZCIT), Perturbações Ondulatórias dos Alísios (POAs) e um caso associado à um centro de baixa pressão, classificado como Circulação Ciclônica. Nos casos de CCM com trovoadas os valores do CAPE+ dos Perfis simulados permitiram classificar a atmosfera como moderadamente instável, enquanto o valor do índice LI representou possibilidade de trovoadas moderadas a trovoadas severas.

Palavras-chave: CCM; Trovoada; Previsão do Tempo.

\section{Mesoscale convective complexes over the Northeast Brazil and adverse phenomena associated}

\begin{abstract}
Forecasting of the Mesoscale Convective Complexes (MCC) is extremely important for the Brazilian Northeast's (BNE) population, as they cause different adverse phenomena such as intense precipitation and thunderstorms causing floods and landslides, provoking social and economic losses. The main purpose of this study is to analyze the MCC development and adverse phenomena associated for the period between 2013 and 2015. The analysis of GOES-13 satellite images in the enhanced infrared channel (IR) were used for the MCC identification. Reanalysis phenomena associated for the period between 2013 and 2015 . The analysis of GOES-13 satellite images in the enhanced infrared channel (IR) were used for the MCC identification. Reanalysis data II of the National Center for Environmental Prediction (NCEP) were used for the synoptic analysis over the streamline fields by the GrADS software. The thermodynamic study was carried
out by the plotting of the simulated vertical profiles, with the analysis based on the LI and CAPE + instability indices. Satellite images of the Tropical Rainfall Measuring Mission (TRMM) and data of the Department of Airspace Control (DECEA) were used for the thunderstorms detection. The precipitation detection was collected from the available automatic and conventional stations of the pluviometric stations network of the National Meteorological Institute (INMET). Sixteen MCC cases were identified during the study period and thunderstorm associated was detected in 6 events. Four different synoptic patterns were associated with the MCC formation: Upper Troposferic Cyclonic Vortex (UTCV), Intertropical Convergence Zone (ITCZ), Wave Perturbation on the trade wind and one case was associated with the low-pressure center, classified as the Cyclonic Circulation. For the MCC events with thunderstorms, the obtained CAPE + values of the simulated vertical profiles made it possible to classify the atmosphere as a moderately unstable, while the LI index value presented the possibility of moderate to severe thunderstorms.
\end{abstract}

Keywords: MCC; Thunderstorm; Weather Forecast.

Topic: Meteorologia, Climatologia e Mudanças Climáticas

Reviewed anonymously in the process of blind peer.

Matheus José Arruda Lyra

Universidade Federal de Alagoas, Brasil

http://lattes.cnpq.br/0276487637942612

http://orcid.org/0000-0003-0890-762X

matheuslyraa@gmail.com

Lucas Carvalho Vieira Cavalcante

Universidade Federal de Alagoas, Brasi

http://lattes.cnpq.br/8282005760215891

http://orcid.org/0000-0002-2258-2270

lucasxave@hotmail.com

\section{Vladimir Levit}

Universidade Federal de Alagoas, Brasil

http://lattes.cnpq.br/1231943260451104

vladimirle@gmail.com

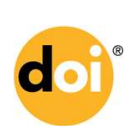

DOI: 10.6008/CBPC2179-6858.2018.003.0008
Received: 10/02/2018

Approved: 24/03/2018
Natalia Fedorova (iD

Universidade Federal de Alagoas, Brasil

http://lattes.cnpq.br/6240947588482690

http://orcid.org/0000-0002-4154-6830

nataliabras@gmail.com
Referencing this:

LYRA, M. J. A.; CAVALCANTE, L. C. V.; LEVIT, V.; FEDOROVA, N.. Complexos convectivos de mesoescala sobre o Nordeste do Brasile fenômenos adversos associados. Revista Ibero Americana de Ciências Ambientais, v.9, n.3, p.95-103, 2018. DOI:

http://doi.org/10.6008/CBPC2179-6858.2018.003.0008 


\section{INTRODUÇÃO}

Os Complexos Convectivos de Mesoescala (CCM) são caracterizados como um conjunto de nuvens Cumulonimbus $(\mathrm{Cb})$, frias e espessas, apresentadas na forma circular e com crescimento vertical explosivo em um intervalo de tempo entre 6 a 12 horas (MADDOX, 1980). O estudo sobre estes sistemas desperta bastante interesse, pois os CCM provocam diversos fenômenos adversos, como precipitações intensas e trovoadas, acarretando em alagamentos, enchentes, deslizamentos de terra e, consequentemente, causam grandes perdas econômicas e sociais. No Nordeste do Brasil, onde há falta de planejamento das cidades, ocupação desordenada de domicílios em encostas e outras áreas de risco, além de afetar a agricultura que é fundamental para a economia da região.

Na região Nordeste, os principais sistemas que provocam precipitação convectiva são: Vórtices Ciclônicos de Altos Níveis (VCAN), Zona de Convergência Intertropical (ZCIT), Ondas de leste (OL), Perturbações Ondulatórias dos Alísios (POAs), Extremidade Frontal (EF), também os Complexos Convectivos de Mesoescala (CCM) e Linhas de Instabilidade (SILVA et al., 2011).

As primeiras pesquisas sobre CCM na região foram feitos por Fedorova et al. (2008) através do estudo da gênese e desenvolvimento destes sistemas através das trajetórias das parcelas de ar geradas a partir do modelo HYSPLIT. Posteriormente, Fedorova et al. (2009) analisaram as características morfológicas dos CCM entre os anos de 1999 e 2009. Albuquerque (2011) verificou os fatores meteorológicos associados ao deslocamento dos mesmos, assim como a influência do El Niño Oscilação Sul (ENOS) em relação à formação. Milhahn (2013) associou a formação dos CCM desta região aos seguintes sistemas sinóticos: Vórtice Ciclônico em Altos Níveis, Extremidade frontal, Zona de Convergência Intertropical e Alísios.

Em relação as trovoadas, o primeiro estudo sobre a atuação deste fenômeno no Estado de Alagoas foi realizado por Brito et al. (2011). Este estudo consistia na análise da frequência das trovoadas entre os anos de 1998 e 2007, e dos processos sinóticos e termodinâmicos associados à sua formação. Com base neste estudo foi criado uma primeira versão do método de previsão (BRITO et al., 2011). Este foi dividido em 3 partes: Climatológica, Sinótica e Termodinâmica. Posteriormente, Cordeiro (2014) realizou um estudo climatológico das trovoadas entre os anos de 1998 e 2012, aperfeiçoando a parte climatológica e sinótica do método de previsão. Mais recentemente, Cavalcante (2016) acrescentou a influência do relevo na formação e intensificação das trovoadas ao método de previsão.

\section{METODOLOGIA}

A área de estudo é compreendida entre as latitudes $0^{\circ}$ e $15^{\circ}$ Sul e longitudes $30^{\circ}$ a $45^{\circ}$ Oeste, sendo esta utilizada na análise da trajetória e desenvolvimento dos CCM selecionados para o estudo. O período de estudo escolhido se estende entre 2013 e 2015. Foram utilizadas imagens do satélite GOES 13 (Geostationary Operational Environmental Satellites) no canal Infravermelho (IR) consultadas no banco de dados imagens no portal da Divisão de Satélites e Sistemas Ambientais (DSA) do Centro de Previsão do Tempo e Estudos Climáticos (CPTEC/INPE). Dados de reanálise II global fornecidos pelo National Centers for 
Environmental Prediction (NCEP) e National Center for Atmospheric Research (NCAR), com resolução de 2,5 de latitude por $2,5^{\circ}$ de longitude. Estes dados de reanálise foram utilizados, através do auxílio do software Grid and Analysis Display System (GrADS) para confecção de campos horizontais de linhas de corrente, os quais foram plotados para os níveis de 925, 850, 700, 500 e 300hPa.

Foram utilizados dados diários de precipitação de estações automáticas e convencionais disponíveis no Banco de Dados Meteorológicos para Ensino e Pesquisa (BDMEP) da rede de estações do Instituto Nacional de Meteorologia (INMET). Os dados foram gerados para cada dia de ocorrência dos CCM nas estações que se encontravam sob a área de influência dos sistemas. A ocorrência de trovoadas foi investigada através do sensor Lightning Imaging Sensor (LIS) do satélite Tropical Rainfall Measuring Mission (TRMM). Este sensor consiste em instrumento usado para detectar relâmpagos nas regiões tropicais do globo. A análise destas ocorrências também foi realizada através de dados do Departamento de Controle do Espaço Aéreo (DECEA).

Os dados de trovoadas foram registrados por Plotagem de METAR, sendo analisados através de códigos SYNOP e METAR do banco de dados de fenômenos adversos das16 estações meteorológicas dos aeroportos dos estados do Nordeste brasileiro. Os eventos foram identificados analisando as imagens do satélite GOES-13 em um intervalo de 1 hora para todos os dias entre os anos de 2013 e 2015 na região de estudo, utilizando as normas propostas por Maddox (1980), conforme a Tabela 1.

Tabela 1: Definição de um CCM proposta por Maddox (1980) baseada em imagens de satélite no canal infravermelho.

\begin{tabular}{|l|l|}
\hline Características físicas & \\
\hline Tamanho: & $\begin{array}{l}\text { A-Cobertura de nuvens com temperaturas } \leq-32^{\circ} \mathrm{C} \text { observadas no canal IR, com área } \geq 100000 \mathrm{~km}{ }^{2} . \\
\text { B }- \text { Região interna da cobertura de nuvens com temperaturas } \leq-52^{\circ} \mathrm{C} \text { observadas no canal IR, com área } \geq \\
50000 \mathrm{~km}^{2} .\end{array}$ \\
\hline Início: & Quando as definições de tamanho A e B sejam satisfeitas. \\
\hline Duração: & As definições de tamanho A e B deverão persistir por um período $\geq 6 \mathrm{~h}$ \\
\hline Extensão Máxima: & Quando a definição do tamanho $\mathrm{A}\left(-32^{\circ} \mathrm{C}\right)$ alcançar seu tamanho máximo. \\
\hline Forma: & Excentricidade $\geq 0,7$ no momento de máxima extensão. \\
\hline
\end{tabular}

Os Perfis verticais simulados (Ps) foram plotados com os dados de reanálise do NCEP através do software OpenGrADs. Estes perfis foram plotados utilizando os 10 níveis padrões (1000, 950, 900, 850, 800, $700,600,500,400$ e $300 \mathrm{hPa}$ ) para o ponto de grade de coordenadas iniciais onde encontrava-se o núcleo dos CCM no seu estágio inicial. O estudo realizado por Cavalcante (2016) mostrou, através de uma comparação entre os perfis simulados (Ps) e as Radiossondagens para Recife/PE, que os Ps plotados com dados do NCEP apresentaram maior representatividade, em relação aos Ps com dados do ECMWF, tanto para as curvas da temperatura do ar e temperatura do ponto d'orvalho quanto para os índices de instabilidade.

Para avaliar a capacidade potencial da atmosfera para o desenvolvimento de trovoadas, foram analisados os Índices de Instabilidade Lifted (LI) e CAPE+ (ALCÂNTARA, 1969). Estudos anteriores avaliaram a eficiência dos índices de instabilidade para estimar a intensidade das trovoadas no estado de Alagoas (BRITO et al., 2011; CORDEIRO, 2014; CAVALCANTE, 2016). Todos os estudos concluíram que os índices que melhor indicaram atmosfera instável ou possibilidade de trovoada foram o índice Lifted (LI) e o CAPE+. 
A CAPE é a área do diagrama SKEW T-LOG P compreendida entre a curva da temperatura do ar e a curva da adiabática suturada. Esta adiabática representa a temperatura de uma parcela de ar que foi levantada a superfície (1000hPa). O CAPE é positivo (CAPE+), também conhecido como energia de flutuabilidade, representa a energia capaz de impulsionar a parcela de ar em seu movimento ascendente. Quanto maior o CAPE+, mais intensa é a convecção, o que implica em um maior desenvolvimento vertical da parcela de ar. Seu valor é utilizado para verificar as condições de instabilidade da atmosfera provocada por esses processos. A tabela 2 apresenta os valores limites de CAPE+, usados como parâmetro para determinar a instabilidade atmosférica.

Tabela 2: Avaliação da instabilidade atmosférica pelo CAPE+.

\begin{tabular}{|l|l|}
\hline Valor do CAPE+ & Estabilidade \\
\hline$<0$ & Estável \\
\hline $0-1000$ & Pouco instável \\
\hline $1000-2500$ & Instabilidade moderada \\
\hline$>2500$ & Forte instabilidade \\
\hline
\end{tabular}

Devido a escolha arbitraria do nível de $850 \mathrm{hPa}$ do indice Showlater, tornou-se díficil de utiliza-lo em casos onde existia uma inversão ou uma rápida queda de umidade passando pela superficie de $850 \mathrm{hPa}$ (ALCÂNTARA, 1969). Por isso, Galway (1956) criou o índice Lifted que é uma modificação do índice Showlater. Nele a parcela de ar é levantada, a partir da superfície (1000hPa), até o nível de 500hPa. O valor deste índice representa a temperatura do ambiente subtraída da temperatura da parcela, ambos no nível de $500 \mathrm{hPa}$. Também pode ser associado a largura do CAPE no nível de 500ha. A tabela 3 apresenta os parâmetros usados para determinar potencial de ocorrência de atividade convectiva através do índice LI desenvolvidos pelo National Weather Service.

Tabela 3: Potencial de ocorrência de atividade convectiva pelo Índice Lifted (LI).

\begin{tabular}{|l|l|}
\hline Índice $\mathrm{LI}$ & Potencial de ocorrência de atividade convectiva \\
\hline $\mathrm{LI}>2$ & Sem atividade convectiva significante \\
\hline $0<\mathrm{LI}<2$ & Possibilidade de ocorrência de convecção rasa \\
\hline$-2<\mathrm{LI}<0$ & Possibilidade de ocorrência de convecção profunda \\
\hline$-4<\mathrm{LI}<-2$ & Possibilidade de chuvas e trovoadas (porém, pouco severas) \\
\hline $\mathrm{LI}<-4$ & Possibilidade de chuvas torrenciais com trovoadas severas \\
\hline
\end{tabular}

$\mathrm{LI}=\mathrm{T}_{500 \mathrm{PPa}}-\mathrm{T}_{500 \mathrm{PPa}}{ }^{\prime}$

$\mathrm{T}_{500 \mathrm{hPa}}=$ temperatura do ar ambiente em $500 \mathrm{hPa}$;

$\mathrm{T}_{500 \mathrm{hPa}}{ }^{\prime}=$ temperatura de uma parcela de ar, na superfície $(1000 \mathrm{hPa})$, levantada até o nível de $500 \mathrm{hPa}$.

\section{RESULTADOS E DISCUSSÃO}

De acordo com a análise das imagens do satélite GOES-13 para o período de estudo (2013 a 2015), foram identificados um total de 16 casos de CCM formados sobre à região Nordeste do Brasil. Cada um dos eventos é apresentado em seu tamanho de extensão máxima na Figura 1, através de imagens no canal Infravermelho realçado.

Fazendo uma comparação entre estudos de anos anteriores foi constatada uma diminuição no número de casos. Albuquerque (2011) e Milhahn (2013) mostraram uma média maior que 7 casos por ano, havendo maior frequência no ano de 2010 e menor frequência em 1999. No período de estudo concentraram-se seis casos em 2013, e outros cinco em 2014 e 2015 cada. Esta diminuição de ocorrências 
pode ser justificada devido ao menor adentramento de periferias frontais na região, estes que são sistemas sinóticos bastante influentes na formação dos CCM, principalmente no Centro-Sul do estado da Bahia.
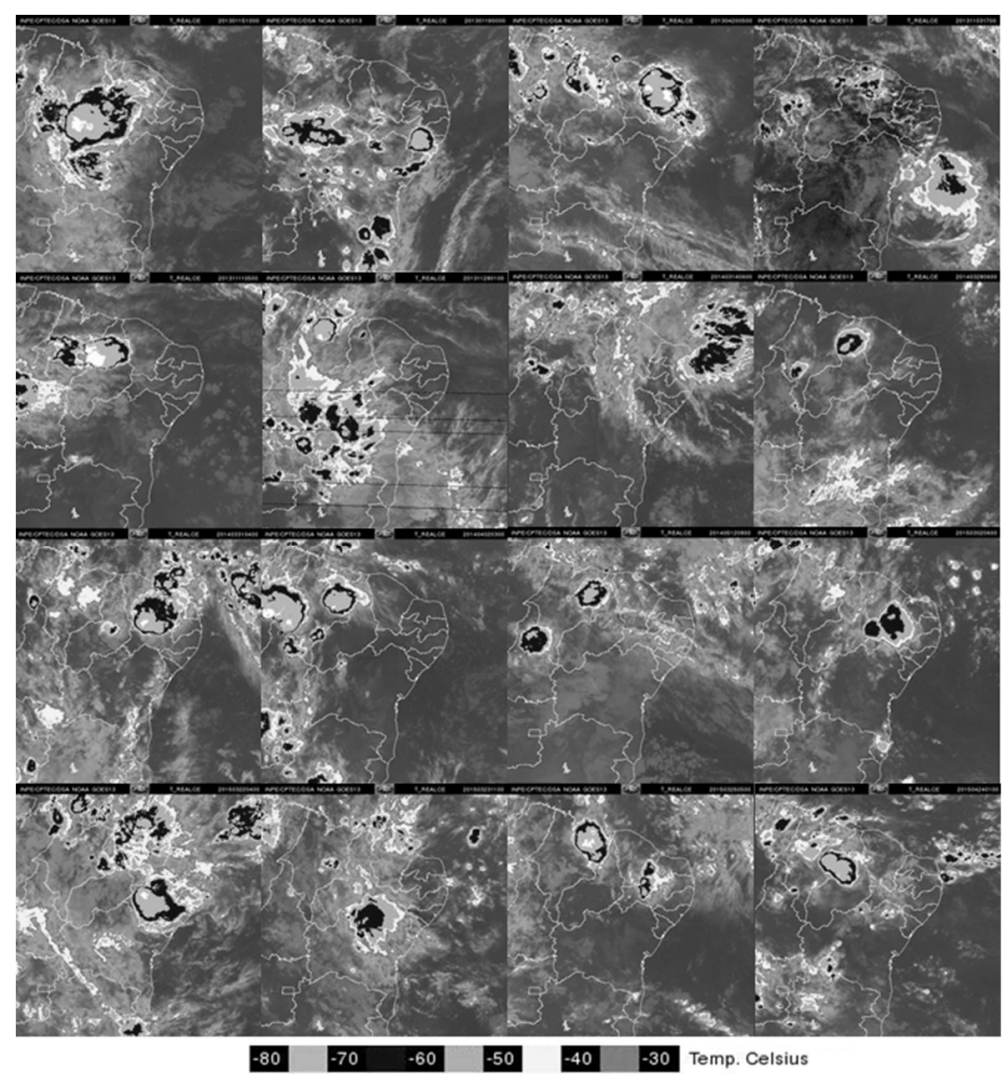

Figura 1: Eventos de CCM identificados dentro do período de análise.

Para analisar de forma eficiente estes casos, observou-se a necessidade do conhecimento da sazonalidade dos mesmos, assim a Figura 2 mostra a distribuição destes casos mensalmente. Os CCM apresentaram maior frequência principalmente no mês de Março. Não havendo ocorrência nos meses de Junho, Julho, Agosto, Setembro, Outubro e Dezembro. Desenvolveram-se com maior frequência durante o outono ( $50 \%$ dos casos), a estação do verão também se apresentou de forma significativa com $31,3 \%$ de ocorrência de CCM, durante a primavera foram constatados apenas $18,7 \%$ dos eventos ocorridos, enquanto no Inverno nenhum caso foi registrado. $O$ desenvolvimento dos CCM durante o verão e outono ocorre devido ao potencial convectivo da região e por estar associado à Zona de Convergência Intertropical que se posiciona mais ao sul durante este período do ano juntamente com a presença dos alísios, aumentando o transporte de umidade, aumento na temperatura da superfície e mais instabilidade favorecendo a atividade convectiva na região.

A partir da análise dos campos de direção e magnitude do vento em diferentes níveis em conjunto com as imagens de satélite, foram identificados padrões sinóticos associados à formação dos eventos de CCM, onde os mesmos encontravam-se dentro da área de circulação dos sistemas sinóticos. (Figura 3). Foram identificados sistemas como Vórtice Ciclônico em Altos Níveis (VCAN), Zona de Convergência Intertropical (ZCIT), Perturbações Ondulatórias dos Alísios (POAs) e um caso associado à um centro de baixa pressão, denominado como Circulação Ciclônica. 


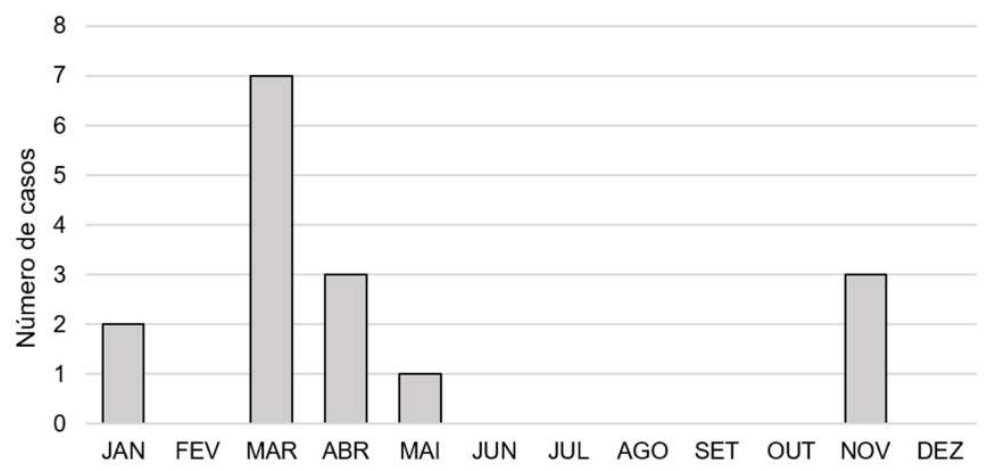

Figura 2: Distribuição mensal da frequência de CCM no NEB entre 2013 e 2015.
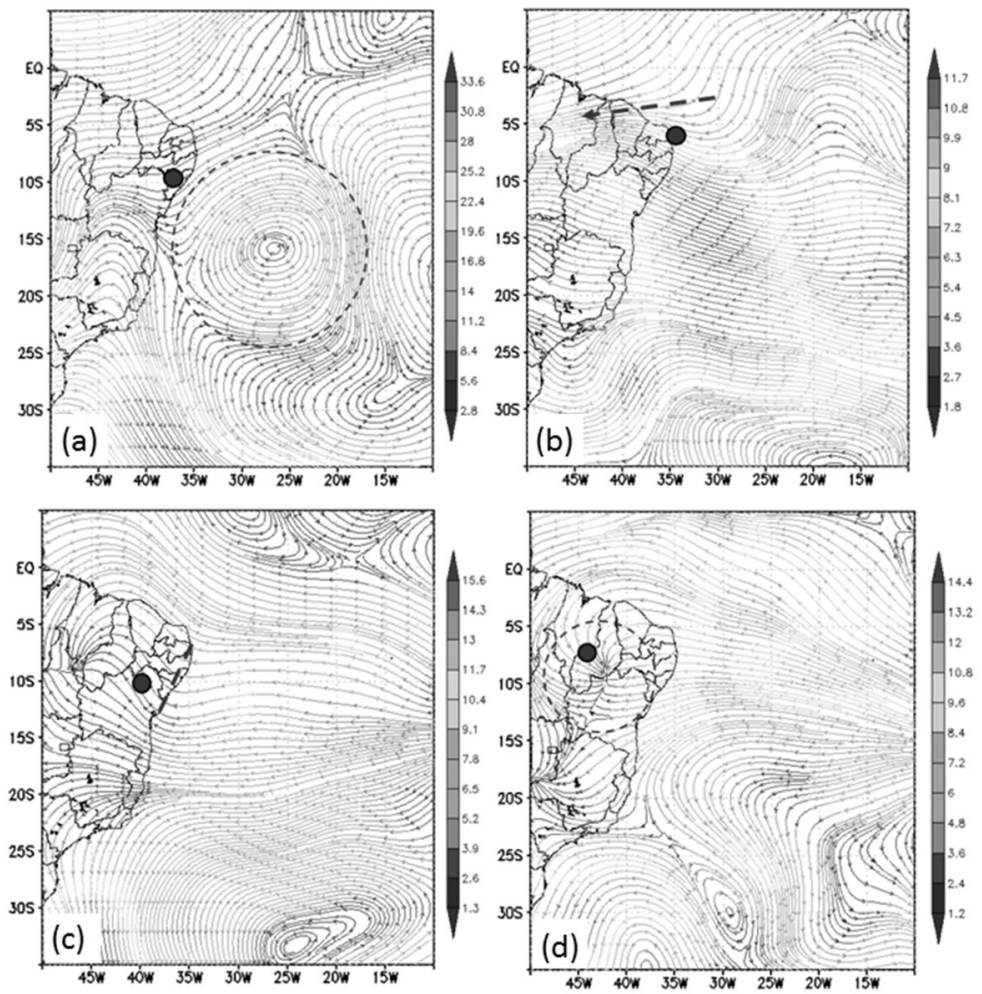

Figura 3: Sistemas sinóticos nas linhas de corrente: VCAN (300hPa (a)), ZCIT (925hPa (b)), POAS (850hPa (c)) e Circulação ciclônica (1000hPa (d)). Dados calculados em metros por segundo $(\mathrm{m} / \mathrm{s})$.

Tabela 4: Principais sistemas sinóticos associados à formação dos CCM para o período em estudo.

\begin{tabular}{|l|l|l|l|}
\hline Data & Sistema Associado & Data & Sistema Associado \\
\hline $15 / 01 / 2013$ & ZCIT & $31 / 03 / 2014$ & ZCIT \\
\hline $19 / 01 / 2013$ & VCAN & $02 / 04 / 2014$ & ZCIT \\
\hline $20 / 04 / 2013$ & ZCIT & $12 / 05 / 2014$ & ZCIT \\
\hline $03 / 11 / 2013$ & VCAN & $02 / 03 / 2015$ & ZCIT \\
\hline $11 / 11 / 2013$ & Circ. Ciclônica & $21 / 03 / 2015$ & ZCIT \\
\hline $27 / 11 / 2013$ & POAs & $23 / 03 / 2015$ & POAs \\
\hline $14 / 03 / 2014$ & VCAN & $24 / 03 / 2015$ & ZCIT \\
\hline $28 / 03 / 2014$ & ZCIT & $24 / 04 / 2015$ & ZCIT \\
\hline
\end{tabular}

Tabela 5: Dados de identificação de trovoadas, com os respectivos estados onde o CCM se formou e se dissipou.

\begin{tabular}{|l|l|l|}
\hline Casos de trovoada registrados & \multicolumn{2}{l|}{} \\
\hline Data & Identificação & Estados \\
\hline $15 / 01 / 2013$ & TRMM & PI-MA \\
\hline $19 / 01 / 2013$ & TRMM & AL-AL \\
\hline $20 / 04 / 2013$ & TRMM & RN-CE \\
\hline $31 / 03 / 2014$ & TRMM & RN-CE \\
\hline $02 / 04 / 2014$ & TRMM & PI-MA \\
\hline $02 / 03 / 2015$ & TRMM & PB-PI \\
\hline
\end{tabular}


Conforme observa-se na Tabela 4, a maior ocorrência de casos esteve associada à circulação ligada a ZCIT, com o maior percentual de ocorrência (62,5\%). Isto ocorre devido ao seu posicionamento mais a Sul da linha do Equador, podendo atingir 4오 de latitude entre os meses de Janeiro e Abril (Ferreira et al., 2005). Em relação ao VCAN, a relação com os CCM ocorreu entre Novembro e Março (18,7\%), devido a maior frequência desse sistema no NEB nesta época do ano. A ocorrência de POAs foi de 12,5\%, e Circulação ciclônica com o menor percentual de ocorrência, associada apenas a um caso (6,3\%). Conforme observado na Tabela 5, entre os 16 casos de CCM analisados no período de estudo, foram registrados 6 eventos de trovoada, os quais foram identificados apenas através das imagens do satélite TRMM.

De acordo com os dados do satélite TRMM (Figura 4), as ocorrências de trovoadas aconteceram com maior incidência nos meses de Janeiro, Março e Abril, apresentando 2 casos em cada mês. As trovoadas se desenvolveram com a mesma frequência no Verão e Outono, onde foram registrados 3 casos (50\%) em ambas estações do ano. Meses esses que são mais propícios a formação de trovoadas nesta região (Cordeiro, 2014).
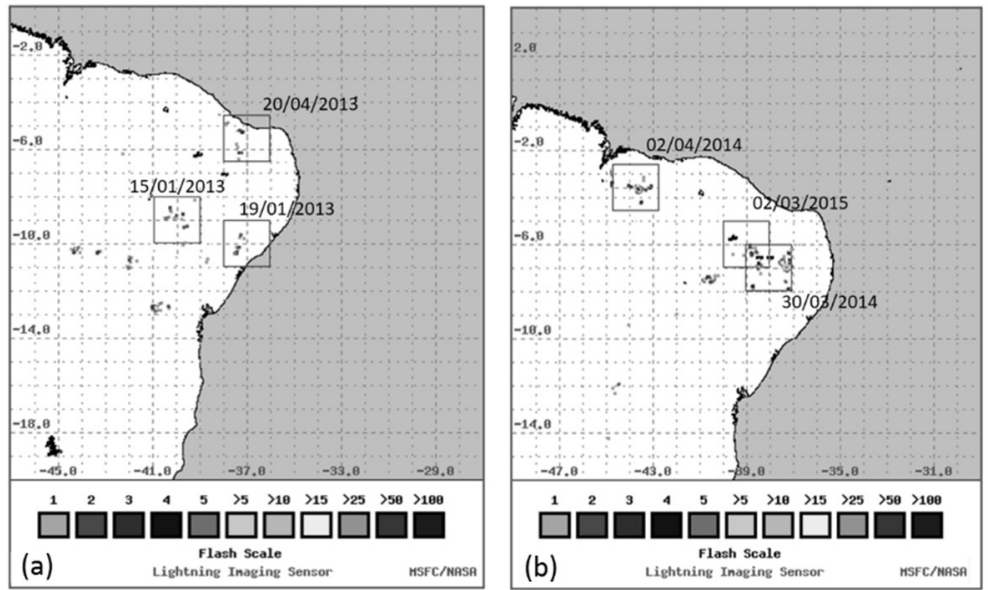

Figura 4: Trovoadas associadas aos CCM identificadas através do sensor LIS do satélite TRMM.

Os valores máximos pluviométricos foram obtidos com o intuito de apresentar os danos causados pelas fortes chuvas associadas aos CCM. Na Tabela 6 são apresentados os valores máximos registrados para os dias onde ocorreram os eventos. Cinco eventos apresentaram valores pluviométricos com mais de $100 \mathrm{~mm}$ em 24 horas. A formação destes CCM está relacionada, em alguns casos com o VCAN e em outros com a ZCIT, sendo estes sistemas responsáveis por precipitação convectiva no Nordeste brasileiro. Estas fortes chuvas, podem ter acarretado desabamento de terra, provocando várias mortes, sendo consideradas de forma negativa. Já as fortes chuvas, também podem ter suas características positivas, como no evento analisado no dia 19 de Janeiro de 2013, na região semiárida do estado de Alagoas, que vivia um longo período de estiagem.

Em relação aos índices de instabilidade dos Ps (Tabela 7), o CAPE+ apresentou valores entre 1000 e $2000 \mathrm{~J} / \mathrm{Kg}$ que permite classificar a atmosfera como moderadamente instável. O caso do dia 20 de Abril de 2013 foi o único com valor inferior a $1000 \mathrm{~J} / \mathrm{kg}$ sendo classificado como instabilidade fraca. O valor do índice LI máximo foi de -3 e mínimo de -6 , que representa possibilidade de trovoadas moderadas $(-4<\mathrm{LI}<-2)$ a trovoadas severas $(\mathrm{LI}<-4)$. 
Tabela 6: Valores de precipitação máxima/24h para os casos de CCM.

\begin{tabular}{|l|l|l|l|}
\hline Data & Precipitação Máx. (24h) & Data & Precipitação Máx. (24h) \\
\hline $15 / 01 / 2013$ & $185 \mathrm{~mm}$ & $31 / 03 / 2014$ & $170 \mathrm{~mm}$ \\
\hline $19 / 01 / 2013$ & $140 \mathrm{~mm}$ & $02 / 04 / 2014$ & $55 \mathrm{~mm}$ \\
\hline $20 / 04 / 2013$ & $180 \mathrm{~mm}$ & $12 / 05 / 2014$ & $75 \mathrm{~mm}$ \\
\hline $03 / 11 / 2013$ & $100 \mathrm{~mm}$ & $02 / 03 / 2015$ & $75 \mathrm{~mm}$ \\
\hline $11 / 11 / 2013$ & $117 \mathrm{~mm}$ & $21 / 03 / 2015$ & $150 \mathrm{~mm}$ \\
\hline $27 / 11 / 2013$ & $62 \mathrm{~mm}$ & $23 / 03 / 2015$ & $75 \mathrm{~mm}$ \\
\hline $14 / 03 / 2014$ & $85 \mathrm{~mm}$ & $24 / 03 / 2015$ & $85 \mathrm{~mm}$ \\
\hline $28 / 03 / 2014$ & $70 \mathrm{~mm}$ & $24 / 04 / 2015$ & $65 \mathrm{~mm}$ \\
\hline
\end{tabular}

Tabela 7: Índices de instabilidade dos Perfis simulados.

\begin{tabular}{|l|l|l|}
\hline Data & CAPE+ & LI \\
\hline 15 de Janeiro de 2013 & 1113 & -3 \\
\hline 19 de Janeiro de 2013 & 1648 & -5 \\
\hline 20 de Abril de 2013 & 865 & -3 \\
\hline 03 de Novembro de 2013 & 1982 & -6 \\
\hline 31 de Março de 2014 & 1415 & -5 \\
\hline 02 de Abril de 2014 & 1117 & -4 \\
\hline
\end{tabular}

\section{CONCLUSÕES}

Foram identificados, de acordo com as normas de Maddox (1980), 16 eventos de CCM no período de Janeiro de 2013 a Dezembro de 2015. Foram associados aos seguintes sistemas sinóticos: VCAN, ZCIT, POAs e Circulação ciclônica. A ZCIT foi constatada com maior frequência (62,5\%). Os CCM analisados apresentaram maiores ocorrência durante o outono e verão, pois o elevado número de registro de casos pode estar associado ao deslocamento da ZCIT que se mantém mais ao sul durante o verão e, principalmente, no outono. Cinco eventos apresentaram valores pluviométricos com mais de $100 \mathrm{~mm}$ em 24 horas, sendo que a formação deste foi associada à circulação do VCAN em alguns casos e em outros com a ZCIT, dando-se estes sistemas responsáveis por precipitação convectiva no Nordeste brasileiro. Os valores do CAPE+ dos Ps permitiram classificar a atmosfera como moderadamente instável, enquanto o valor do índice LI representa possibilidade de trovoadas moderadas a trovoadas severas.

\section{REFERÊNCIAS}

ALBUQUERQUE, C. S. M.. Desenvolvimento e Trajetórias dos Complexos Convectivos de Mesoescala no Estado de Alagoas Entre os Anos de 1999 e 2009. Monografia (Bacharelado em Meteorologia) - Universidade Federal de Alagoas, 2011.

ALCÂNTARA, F.. Manual de análise do Diagrama 'Skew-T, log P'. Ministério da Aeronáutica, 1969.

BRITO, B. M.; LEVIT, V.; FEDORAVA, N.; MOLION, L. C. B.; TENÓRIO, R. S.; RODRIGUES, R. N.; SILVA, B. F. P.. Análise do comportamento das trovoadas no Estado de Alagoas, previsão a curto prazo. Revista Brasileira de Meteorologia, Maceió, v.26, n.2, p.243-256. 2011. DOI:

http://dx.doi.org/10.1590/S0102-77862011000200009.

CAVALCANTE, L. C. V.. Análise dos eventos com trovoadas no estado de Alagoas entre 2013 e 2015, levando em conta a influência do relevo. Monografia (Bacharelado em Meteorologia) - Universidade Federal de Alagoas, 2016.
CORDEIRO, E. S.. Analise sinótica dos eventos com trovoadas para o estado de Alagoas dentre o período de 15 anos (1998-2012). Dissertação (Mestrado em Meteorologia) - Universidade Federal de Alagoas, 2014.

FEDOROVA, N.; LEVIT, V.; RODRIGUES, L. R. L.; COSTA, S. B.. Mesoscale Convective Complex Genesis and forecast in Alagoas State of Brazil. Journal of the Georgian Geophysical Society, v.12, p.36-44, 2008.

FEDOROVA, N.; LEVIT, V.; SILVEIRA, M. H. S.; SILVA, B. F. P.; AMIRANASHVILI, A. G.. Mesoscale Convective Complexes on the Northeastern Coast of Brazil. Journal of the Georgian Geophysical Society, v.13, p.36-49, 2009.

FERREIRA, G. A.; MELLO, N. G. S.. Principais Sistemas Atmosféricos Atuantes Sobre a Região Nordeste do Brasil e a Influência dos Oceanos Pacífico e Atlântico no Clima da Região. Revista Brasileira de Climatologia, v.1, n.1, p.15-28, 2005. 
GALWAY, J. G.. The lifted index as a predictor of latent instability. Bulletim of the American Meteorology Society, v.37, n.10, p.528-529, 1956

MADDOX, R. A.. Mesoscale Convective Complexes. Bulletin of the American Meteorological Society, v.61, n.11, p.1374$1387,1980$.

MILHAHN, A. W.. Correntes de jato e sistemas sinóticos associados aos Complexos Convectivos de Mesoescala no
Nordeste Brasileiro. Monografia (Bacharelado em Meteorologia) - Universidade Federal de Alagoas, 2013.

SILVA, B. F. P.; FEDOROVA, N.; LEVIT, V.; BRITO, B. M.; PERESETSKY, A.. Sistemas sinóticos associados às precipitações intensas no Estado de Alagoas. Revista Brasileira de Meteorologia, v.26, n.3, p.295-310, 2011. DOI: http://dx.doi.org/10.1590/S0102-77862011000300001

A CBPC - Companhia Brasileira de Produção Científica (CNPJ: 11.221.422/0001-03) detém os direitos materiais desta publicação. Os direitos referem-se à publicação do trabalho em qualquer parte do mundo, incluindo os direitos às renovações, expansões e disseminações da contribuiç̃ão, bem como outros direitos subsidiários. Todos os trabalhos publicados eletronicamente poderão posteriormente ser publicados em coletâneas impressas sob coordenação da Sustenere Publishing, da Companhia Brasileira de Produção Científica e seus parceiros autorizados. Os (as) autores (as) preservam os direitos autorais, mas não têm permissão para a publicação da contribuição em outro meio, impresso ou digital, em português ou em tradução. 